\title{
Analysis of DoS Attacks in Delay Tolerant Networks for Emergency Evacuation
}

\author{
Philip Asuquo, Haitham Cruickshank, \\ Institute for Communication Systems \\ $5 \mathrm{G}$ Innovation Centre \\ University of Surrey, UK \\ Email: p.asuquo@surrey.ac.uk
}

\author{
Zhili Sun, Ganesh Chandrasekaran \\ Institute for Communication Systems \\ $5 \mathrm{G}$ Innovation Centre \\ University of Surrey, UK \\ Email: h.cruickshank@surrey.ac.uk
}

\begin{abstract}
In the event of a disaster, there is a severe damage/destruction to physical infrastructures such as telecommunication and power lines which result in the disruption of communication in this areas. For such scenarios, Delay Tolerant Network (DTN) provides an alternative means of communication. In Delay Tolerant Networks (DTNs), a message from a source node may be delivered to the destination node despite the nonexistence of an infrastructure and an end-to-end connectivity. However DTNs are susceptible to security threats such as DoS attacks targeted at disrupting relayed packets or dropping critical packets during a disaster rescue operation. DoS attacks consist of blackhole, grayhole, wormhole, packet flooding attacks etc. The scope of this paper is to study the impacts of blackhole and packet flooding attacks in a post disaster communication network using DTN. Various performance metrics in DTN have been used to study the impacts of different DoS attacks in DTN and a comprehensive analysis is presented.
\end{abstract}

Keywords-Delay Tolerant Networks, Denial of Service attacks, blackhole, grayhole, wormhole.

\section{INTRODUCTION}

In disaster scenarios, emergency support communication can be set up using Delay/Disruption Tolerant Network(DTN) to facilitate rescue and relief operations [1].In the event of a disaster, there are damages and destruction to the telecommunication infrastructure which result in the disruption of communication links. Recovery of this infrastructure under natural disasters take a long time as a result of the extent of these damages and lack of preparedness or experience in these situation [2]. In the Great East Japan earthquake, it took over a month to restore damaged telecommunication infrastructures in the areas that were affected by disaster [3]. Recently, there have been disasters which have shown the impact they can have on the economy and the people such as the Boko Haram insurgency in Nigeria [4], Hurricane Katrina,earthquake in Haiti,Tsunami in Japan and the 9/11 terrorist attack [5]. Communication infrastructures with fragile connectivity have no security solutions from the current Internet Protocols today, however much effort has been made by the academia and research groups towards achieving an efficient and effective communication model for networks with no end-to-end connectivity and variable long delays [6]. DTN is considered as an effective method of communication in areas requiring emergency communication support [7] however [3] points out that the mobility modeling used by DTNs are not suitable for disaster response communication networks as a result of its mobility pattern which are suitable for a disaster scenarios. Recent work in rescue operations demonstrates the use of DTN in information gathering on disaster victims and sharing among rescue workers [8], another work (MONAC) [9] focuses on information disseminated by victims using twitter messages through a DTN Network which is characterised by a variable long delay and there is no guarantee of delivery.

The remainder of the paper is structured as follows, Section II gives a brief description of related work carried out on disaster recovery and resiliency. In Section III, we look at DoS attacks in DTN and classify the different types of DTN attacks. Section IV describes our system model and architecture which includes our threat model, simulation setup and the results and discussions. While in Section V, we give a brief summary of our analysis on DoS attacks in DTN.

\section{RELATED WORK}

In large scale disasters where the telephony services are damaged or destroyed such as the recent North Indian Ocean flooding and the 2011 Tohoku earthquake were the power and communication lines were unavailable for a long time, DTNs were used as a result of their independency from fix infrastructures and resiliency [10]. The IRTF InformationCentric Networking Research Group (ICNRG) Internet draft on ICN Baseline Scenario [11] recommends three post disaster mobility models for emergency recovery operations. The first model focuses on victims and disaster responders after a disaster has occurred by extracting the key mobility patterns used by response services to model a post disaster mobility traffic pattern, while the second model focuses on emergency responders using Random Way Point(RWP) with attraction points and a more realistic traffic modeling systems. The third model concentrates on the mobility patterns using video traffic over MANET with the integration of the emergency overlay protocol into the DTN architecture using the ferry transport scheme. There are many approaches in DTN to provide connectivity, one of them is using a controllable mobility pattern with a message ferry scheme to provide connectivity [12]. The process of evacuating disaster victims from the incident area after a disaster requires the use of a realistic mobility contact pattern. A related literature in crisis management investigates a worst case scenario with a few mobile users available, they evaluate the performance of Epidemic and HiBOp with special interest in the overhead ratio, latency and message loss rate. Their evaluation results show that multi-copy routing 
protocols perform better than flooding protocols like Epidemic when the message generated by the node is more than what the DTN can accommodate based on the size of the buffer and the bandwidth available [13]. Vehicular Ad-hoc Networks (VANET) and Vehicular Delay Tolerant Networks (VDTN) are also used to provide emergency support in disaster scenarios. The use of hybrid DTN as an auxiliary mechanism in the distribution of warning messages from a control centre for public safety is proposed by [14]. Similarly, the gathering of information from the public to the control centre with an emergency routing scheme which is a directional-based routing protocol via vehicles to vehicles is proposed by [15].

\section{DENIAL OF SERVICE (DoS) ATTACKS IN DTNS}

The application of DTN is limited as a result of security and privacy issues associated with it. Just like other wireless sensor networks, DTN have similar threats and vulnerabilities [1]. The presence of malicious nodes in an emergency response network pose serious threats in data dissemination during the evacuation process. Traditional Mechanisms such as the Public Key Infrastructure(PKI) and Certificates Revocation List(CRL) are not suitable for DTN as a result of its lack of infrastructure and intermittent connectivity [16]. Detection and mitigation schemes in other networks are not applicable in DTN as a result of its unique characteristics. A study carried out by [17] on the efficiency of DTN without a security mechanism shows that DTN is very vulnerable to DoS Attacks. DoS attacks in DTN include packet dropping attacks, bundle flooding, wormhole attacks. Researchers have focused more on improving routing schemes in DTN than making security provisions for disconnected networks or networks that lack end-to-end connectivity.

1) Packet Dropping Attacks in DTNs: Some Authors [18], [19] have addressed packet dropping attacks in DTNs as blackhole, grayhole and routing misbehaviour of nodes. These attacks are carried out by compromised nodes with forged metrics that have the tendencies to drop all (blackhole attack) or a certain percentage (grayhole attack) of the messages to be forwarded. There has been a lot of approaches to mitigating blackhole and grayhole attacks, experimental simulations and theoretical analysis of existing research works show the degradation of the network when nodes are compromised with malicious behaviours. Simulations carried out on the impact of blackhole and grayhole attacks show a significant decrease in the message delivery as the number of malicious nodes increase in the network and an increase in the latency of the network [18].

2) Bundle Flooding: DTNs are susceptible to flooding attacks as a result of the limitation in buffer size and bandwidth, malicious nodes can flood the network with many packets which can degrade the network performance and prevent legitimate packets from reaching their destination nodes [23]. Flooding attacks consume scare resources as nodes will transmit and receive malicious packets flooded in the network. With valid cryptographic credentials, flooding attacks carried out by outside attackers can easily be detected using valid signatures. When considering internal attacks such as flooding attacks, authentication of packets does not guarantee delivery to the destination nodes thus making bundle flooding in DTN an open issue [20]. A few techniques [21], [22] have been proposed to mitigate flooding attacks in DTNs.

3) Wormhole Attacks in DTNs: Wormhole attacks were first introduced by [23] in wireless networks, these attacks are recorded in one area of the network and replayed at another segment of the network by adversary nodes [24]. In MANET and WSNs, wormhole attack was previously seen as a type of routing misbehaviour [25]. Packets flowing through a wormhole can be selectively dropped or delayed by adversary nodes to degrade the network performance of a wireless network. As these packets are re-routed and replayed in different areas of the network, the security mechanisms are not compromised which makes detection difficult using cryptographic verification schemes.

\section{System Model AND ARChitecture}

In post disasters, the availability of a reliable emergency communication network is very important to ensure an effective rescue and evacuation process. DTNs provide a means of communication in the non-availability or destruction of communication infrastructure. There are other disruptions that can degrade the network and disrupt effective communications in rescue operations. These disruptions include routing misbehaviour and network attacks. We investigate the resiliency of an emergency communication network under two categories of DoS attacks. We use the Post-disaster mobility model 1 [7] recommended by IETF for ICN Baseline Scenarios on Emergency Support and Disaster Recovery in DTN [10]. We analyse the effect of these attacks on the performance of the Emergency communication network during the evacuation process and rescue operation.

We assume there is a DTN gateway which provides connectivity via a geostationary satellite while the telecommunication infrastructure is down and messages are forward hop-by-hop through DTN enabled nodes as shown in fig 1. For clarity, we assume that all the messages have the same predefined size. It is still very impractical to allow unlimited delays, therefore we assume each packet has a lifetime and will be discarded when its Time-To-Live (TTL) expires. The relief operation begins after the disaster, Casualty Collection Points, Temporary Care Centre and the Hospital are declared as centres that take part in the evacuation process. These centres are responsible for rescue and relief operations which involves movement of victims from the incident area to the evacuation point, giving first aid treatment to victims with immediate medical attention.

\section{A. Post Disaster Communication Network}

In our work, we assume that there are four on-site locations and five groups of on-site actors(responders) within the emergency rescue area. We use the ETSI Emergency reference scenario for deploying emergency communication TS-103-260 1 and 2 which covers a Mass Transport Accident Scenario (MTA) and an Earthquake Scenario as our baseline for rescue and relief operation [26].

1) On-Site Locations: In figure 2, a brief description of the disaster relief operation from the incident area to the hospital which is assumed as the final evacuation point is presented. 


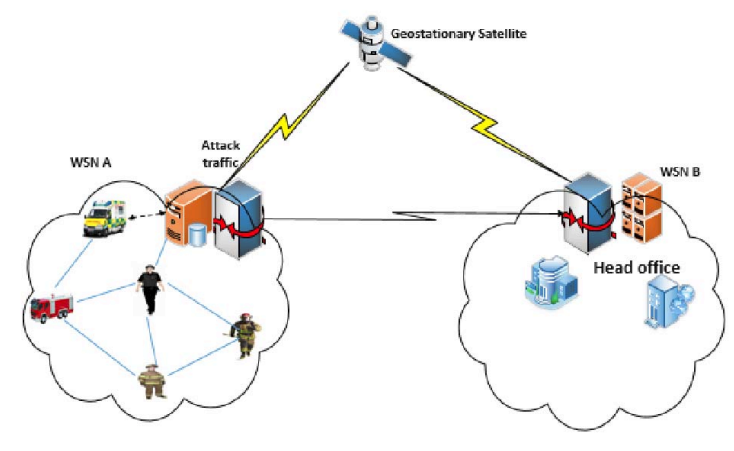

Fig. 1: An Emergency Communication Network

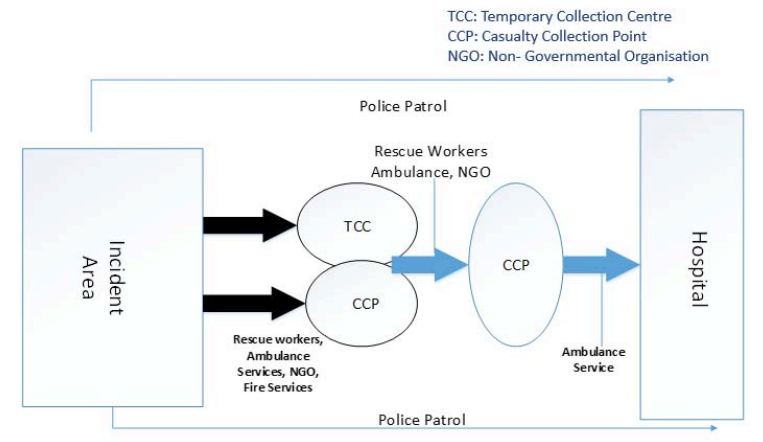

Fig. 2: On-site actors and locations for Disaster Relief Operations

a) Incident Area: This is the area with obvious or supposed threats to physical/psychological health, properties and environment. The emergency responders in the incident area alert and provide initial accident description to the other on-site locations.

b) Casualty Collection Point/Temporary Care Centre $(C C P / T C C)$ : This area is the holding area, disaster victims are moved from the incident area to the CCP/TCC area where victims that need immediate medical attention are taken care of in the TCC and the others moved to the CCP en-route the hospital and final evacuation as shown in figure 2.

c) Casualty Collection Point $(C C P)$ : The $\mathrm{CCP}$ is the second holding area that serves as an intermediary hub for the transportation of victims to emergency medical centre (Hospital).

d) Hospital: The hospital serves as an immediate emergency medical Centre and the final evacuation hub for the disaster relief operations.

\section{2) On-Site Actors/Roles:}

a) Rescue Workers: Rescue workers transport victims to CCP/TCC, hand over victims to responders in the CPP/TCC and return to incident area. They obtain alert and initial accident description and forward it to other locations. The Rescue workers also form part of responders on other locations. b) Ambulance Service: Triage and treatment of the injured victims and organising medical evacuation from the incident area to the hospital.

c) Police: Responsible for guarding the hazard area and public order.

d) Fire Services: Securing the hazard areas, fighting fires and searching for disaster victims.

e) NGOs: These are voluntary organisations with a wide range of roles assisting the public service which are in place.

\section{B. Threat Model}

We present an adversary model which is similar to the one presented in [19], [27]. We consider two types of DoS attacks, packet dropping is our primary attack while flooding attacks is our secondary attack. The secondary attack is initiated when some emergency responder nodes drop packets, they create additional packets and forward them to other nodes participating in the evacuation process. Detection and Mitigation schemes are very crucial for the survivability of an intermittently connected network. A successful DoS attack in a DTN Emergency communication network will degrade, disrupt and disable the DTN nodes that participate in the recovery and evacuation process of a disaster relief operation. For both blackhole and grayhole attacks, we consider 10-50 percent of the emergency responders to be malicious adversaries.

\section{Simulation Setup}

To evaluate the performance of DTN under DoS attacks, we carried out a set of simulations in the Opportunistic Network Environment simulator (ONE) [28], the ONE has been specifically developed for evaluating DTN application protocols and routing. In our experiments, we evaluate both single copy and multiple copy routing protocols. We considered a small scale disaster scenario using our campus map (University of Surrey)as the experimental test bed. Five groups of responders make up the emergency rescue team and detailed information on the simulation parameters is presented in Table 1

TABLE I: Simulation Parameters

\begin{tabular}{|l|l|}
\hline Scenario End Time & 6 hours \\
\hline No of Nodes & 100 \\
\hline TTL (minutes) & 180 \\
\hline No of Groups & $\begin{array}{l}5 \text { [Rescue workers - 50, Patrol team - 10, NGO - } \\
10, \text { Ambulance- 20, Fire Service 20] }\end{array}$ \\
\hline Area & University of Surrey, Guildford, UK. \\
\hline Buffer Size & $50 \mathrm{MB}$ \\
\hline $\begin{array}{l}\text { Group } \\
\text { Speed(Km/h) }\end{array}$ & $0.5-1.5$ (Pedestrians) and 2.7-13.9 (Vehicles). \\
\hline
\end{tabular}

The following performance metrics are considered in analysing the impact of the DoS attacks in Emergency scenarios using DTNs

f) Delivery Ratio: - This is the proportion of messages that have been delivered out of the total unique messages created.

g) Overhead Ratio: - This is the transmission cost incurred in delivering messages across the network. It is the total number of messages transmitted in the simulation divided by the total number of messages created. 
h) Latency: - This is the measure of the average delay in the network. The latency is the time between when a message is created and when the message is received at a destination.

\section{Results and Discussions}

We investigated the impacts of DoS attacks on message delivery in a post disaster scenario. Our results show the effects of increasing the number of malicious nodes in the network. Fig 2 shows the effects of blackhole attacks on the message delivery. There was a significant drop in the message delivery ratio as the number of attackers increased, there was a reduction in the number of messages successfully relayed to the intended emergency responders.

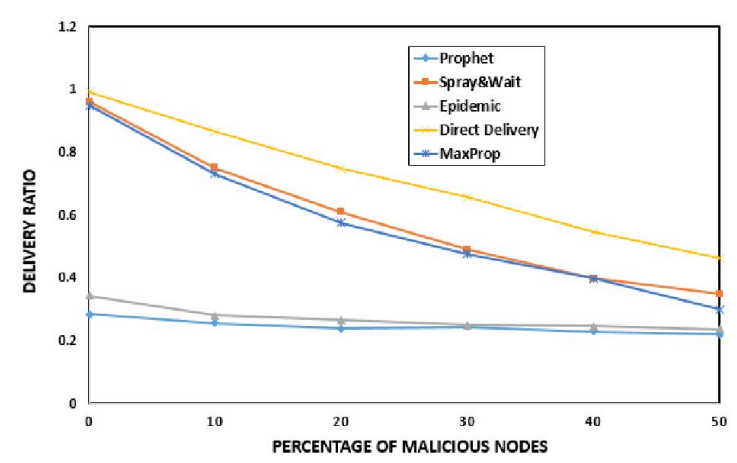

Fig. 3: Impact of Blackhole attacks on Message Delivery

In fig 3, the average delay of the messages sent across the emergency network is presented. Considering internal attacks, there is a trade-off between message delivery ratio and message delay. When the percentage of malicious nodes in the network increases, a message that is not delivered with a longer delay is more likely to be dropped and the dropped message is not considered in calculating the message latency. We also observed similar results in [29] investigating the impact of message delivery and latency and [18] analysing the impact of blackhole attacks on message delivery ratio and latency in DTN routing. However, the Direct Delivery routing protocol has a higher lantency ratio as a result of the time a message spends in its buffer before encountering the destination node.

Fig 4 shows the message overhead ratio of the network, the flooding based routing protocols Epidemic and Prophet considered in this evaluation have the highest overhead ratio in the network with Epidemic higher than Prophet. This result suggest that since both protocols are multi-copy routing protocols that flood packets, Prophet has a smaller overhead because it makes use of a probabilistic metric that determines the forwarding decision to replicate a message to an encountered node while Epidemic is a naive replication based routing protocol. Direct Delivery, Spray and Wait and MaxProp have lower overheads since their forwarding decisions are based on contact with destination node in Direct Delivery and Spray and Wait, while MaxProp assigns priority to new packets and prevents reception of the same packet by discarding it.

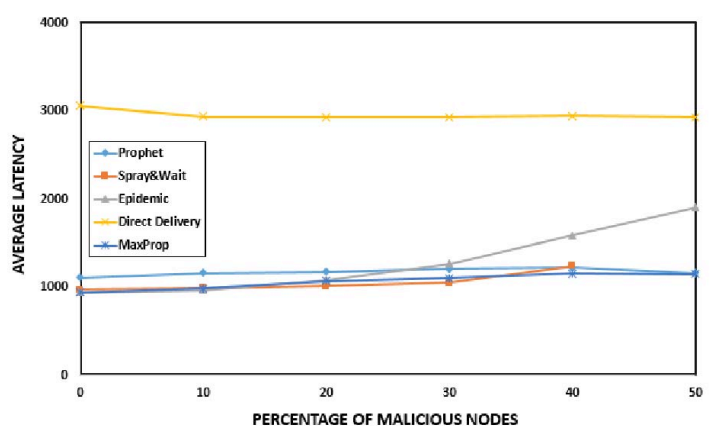

Fig. 4: Impact of Blackhole attacks on Latency

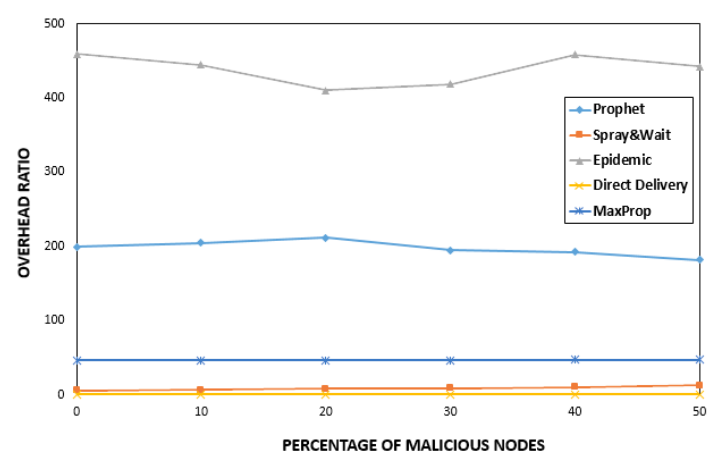

Fig. 5: Impact of blackhole attacks on overhead ratio

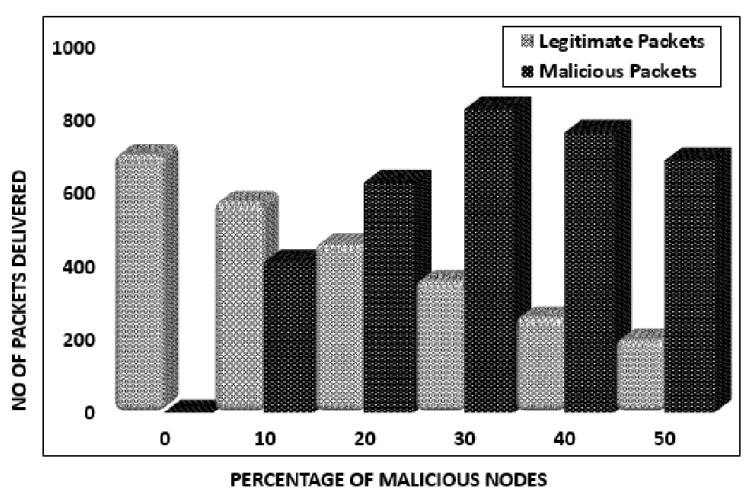

Fig. 6: Legitimate vs malicious packets in Direct Delivery

In fig 5, 6 and 7, we show the impact of the secondary attack on the Emergency communication network, the number of legitimate packet decreases as more adversary nodes take part in the packet flood attack while the number of malicious messages delivered to destination nodes increases. This type of attack is more detrimental to the evacuation process as malicious messages may be aimed at fraudulent activities such as over exaggerating the number of victims or overstated needs like requesting for more ambulance services. 


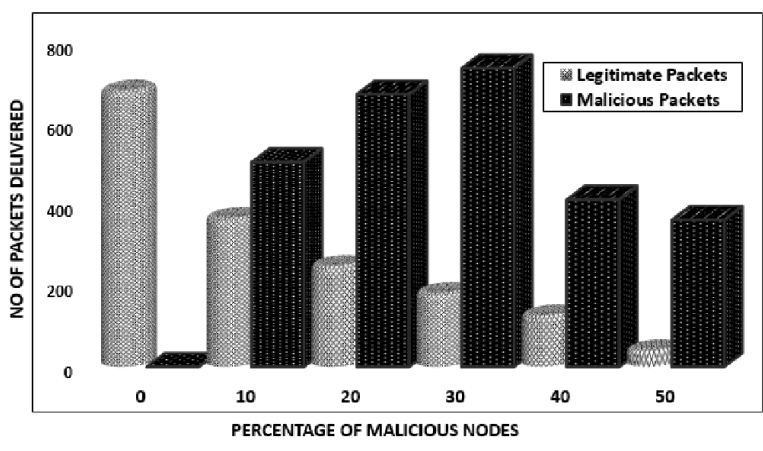

Fig. 7: Legitimate vs malicious packets in Spray and Wait

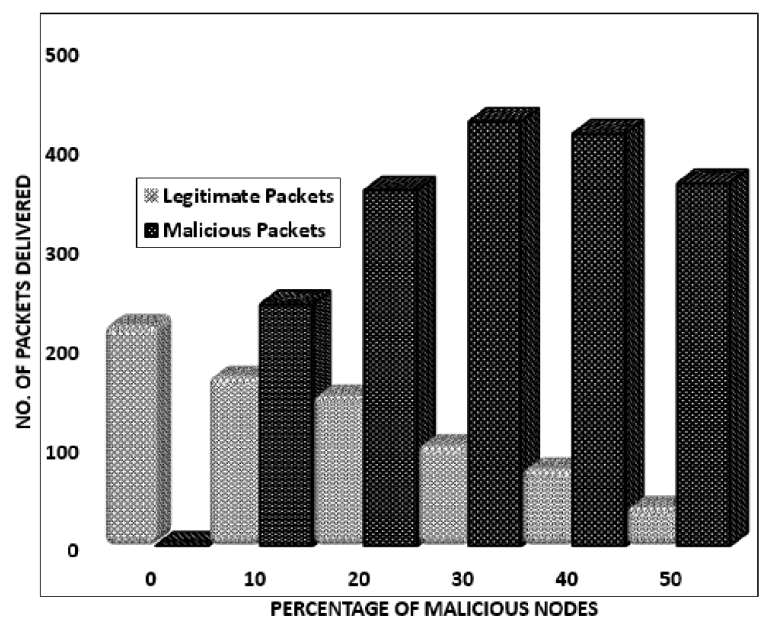

Fig. 8: Legitimate vs malicious packets in Epidemic

\section{CONCLUSion AND Future Work}

In this paper, a comprehensive study of the impacts of DoS attacks including blackhole, and packet flooding attacks in an emergency communication network using DTN has been presented. Comparisons using different performance metrics for the different DoS attacks gives a clear insight of some of the impacts of DoS attacks in DTNs. Apart from network degradation, compromised emergency responders that participate in disaster recovery and evacuation can drop high priority messages relayed to them which could be request for immediate services at the incident area such as requesting for more fire fighters or ambulance services as a result of the increase in the number of casualties. In our future work, we will design and implement a collaborative trust management protocol with an integrated buffer management scheme for mitigating routing misbehaviours in DTNs.

\section{ACKNOWLEDGMENT}

The funding of this work is from the Tertiary Education Trust Fund (TETFUND) of the Federal Government of Nigeria. The authors wish to acknowledge the support of the Institute for Communication Systems (ICS), Home of the 5G Innovation Centre, University of Surrey, Guildford, United Kingdom

\section{REFERENCES}

[1] T. de Oliveira, S. de Oliveira, D. Macedo, and J Nogueira, "An adaptive security management model for emergency networks," in Network Operations and Management Symposium (LANOMS), 2011 7th Latin American, Oct. 2011, pp. 1-4.

[2] M. Huda, F. Yasmeen, S. Yamada, and N. Sonehara, "An approach for short message resilience in disaster-stricken areas," in 2012 International Conference on Information Networking (ICOIN), Feb. 2012, pp. 120-125.

[3] R. Yamashita and K. Takami, "Safety information gathering via information carriers through a DTN in a disaster-stricken area," in 2013 International Conference on ICT Convergence (ICTC), Oct. 2013, pp. 429-434.

[4] I. Mantzikos. (2013, October) Boko haram - anatomy of crisis. ISSN 2053-8626. International Relations. Bristol, Uk.

[5] M. Abbas, N. Yusof, and N. Fisal, "Performance evaluation of Binary Spray and Wait OppNet protocol in the context of emergency scenario," in 2013 IEEE International Conference on Pervasive Computing and Communications Workshops (PERCOM Workshops), Mar. 2013, pp. 517-522.

[6] M. Shanmugam, S. Thiruvengadam, C. Cordier, and J.-P. Le Rouzic, "Emergency Services in Delay Tolerant Environments," in Vehicular Technology Conference, 2006. VTC-2006 Fall. 2006 IEEE 64th, Sep. 2006, pp. 1-5.

[7] M. Uddin, D. Nicol, T. Abdelzaher, and R. Kravets, "A post-disaster mobility model for Delay Tolerant Networking," in Simulation Conference (WSC), Proceedings of the 2009 Winter, Dec. 2009, pp. 2785-2796.

[8] R. Shah, S. Roy, S. Jain, and W. Brunette, "Data MULEs: modeling a three-tier architecture for sparse sensor networks," in 2003 IEEE International Workshop on Sensor Network Protocols and Applications, 2003. Proceedings of the First IEEE, May 2003, pp. 30-41.

[9] S. George, W. Zhou, H. Chenji, M. Won, Y. O. Lee, A. Pazarloglou, R. Stoleru, and P. Barooah, "DistressNet: a wireless ad hoc and sensor network architecture for situation management in disaster response," IEEE Communications Magazine, vol. 48, no. 3, pp. 128-136, Mar. 2010

[10] E. K. Pentikousis, "Information-centric networking: Baseline scenarios onemergency support and disaster recovery," Internet Draft RFC 7476, Tech. Rep., 2014.

[11] E. Davies, G. Tyson, B. Ohlman, S. Eum, A. Molinaro, D. Corujo, K. Pentikousis, and G. Boggia, "Information-centric Networking: Baseline Scenarios," Tech. Rep.

[12] M. C. Chuah and W.-B. Ma, "Integrated Buffer and Route Management in a DTN with Message Ferry," in IEEE Military Communications Conference, 2006. MILCOM 2006, Oct. 2006, pp. 1-7.

[13] B. Raffaele, C. Marco, and P. Andrea, "Opportunistic networking overlays for ICT services in crisis management," Proceedings of the 5th International ISCRAM Conference Washington, DC, USA, May 2008, 2008.

[14] D. Camara, C. Bonnet, and F. Filali, "Propagation of Public Safety Warning Messages: A Delay Tolerant Network Approach," in 2010 IEEE Wireless Communications and Networking Conference (WCNC), Apr. 2010, pp. 1-6.

[15] R. Namritha and K. Karuppanan, "Opportunistic dissemination of emergency messages using VANET on urban roads," in 2011 International Conference on Recent Trends in Information Technology (ICRTIT), Jun. 2011, pp. 172-177.

[16] S. Farrell and V. Cahill, "Security considerations in space and delay tolerant networks," in Second IEEE International Conference on Space Mission Challenges for Information Technology, 2006. SMC-IT 2006, 2006, pp. 8 pp. -38 .

[17] F. C. Choo, M. C. Chan, and E.-C. Chang, "Robustness of DTN against routing attacks," in 2010 Second International Conference on Communication Systems and Networks (COMSNETS), Jan. 2010, pp. $1-10$. 
[18] I.-R. Chen, F. Bao, M. Chang, and J.-H. Cho, "Dynamic Trust Management for Delay Tolerant Networks and Its Application to Secure Routing," IEEE Transactions on Parallel and Distributed Systems, vol. 25, no. 5, pp. 1200-1210, May 2014.

[19] H. Zhu, S. Du, Z. Gao, M. Dong, and Z. Cao, "A Probabilistic Misbehavior Detection Scheme toward Efficient Trust Establishment in DelayTolerant Networks," IEEE Transactions on Parallel and Distributed Systems, vol. 25, no. 1, pp. 22-32, Jan. 2014.

[20] I. Parris and T. Henderson, "Friend or Flood? Social Prevention of Flooding Attacks in Mobile Opportunistic Networks," in 2014 IEEE 34th International Conference on Distributed Computing Systems Workshops (ICDCSW), Jun. 2014, pp. 16-21.

[21] Q. Li, W. Gao, S. Zhu, and G. Cao, "To Lie or to Comply: Defending against Flood Attacks in Disruption Tolerant Networks" IEEE Transactions on Dependable and Secure Computing, vol. 10, no. 3, pp. 168-182, May 2013.

[22] G. Ansa, H. Criuckshank, Z. Sun, and M. Al-Siyabi, "A DOS-resilient design for delay tolerant networks," in Wireless Communications and Mobile Computing Conference (IWCMC), 2011 7th International, Jul. 2011, pp. 424-429.

[23] Y.-C. Hu, A. Perrig, and D. Johnson, "Packet leashes: a defense against wormhole attacks in wireless networks," in INFOCOM 2003. Twenty-Second Annual Joint Conference of the IEEE Computer and Communications. IEEE Societies, vol. 3, Mar. 2003, pp. 1976-1986 vol.3.

[24] C. Karlof and D. Wagner, "Secure routing in wireless sensor networks: attacks and countermeasures," in 2003 IEEE International Workshop on Sensor Network Protocols and Applications, 2003. Proceedings of the First IEEE, May 2003, pp. 113-127.

[25] P. Lee, A. Clark, L. Bushnell, and R. Poovendran, "A Passivity Framework for Modeling and Mitigating Wormhole Attacks on Networked Control Systems," IEEE Transactions on Automatic Control, vol. 59, no. 12, pp. 3224-3237, Dec. 2014.

[26] "ETSI - ETSI New Specifications on Scenarios for Emergency Communications in Disasters." [Online]. Available: http://www.etsi.org/news-events/news/970-2015-06-press-etsi-newspecifications-on-scenarios-for-emergency-communications-in-disasters

[27] Y. Guo, S. Schildt, T. Pougel, S. Rottmann, and L. Wolf, "Mitigating Blackhole attacks in a hybrid VDTN," in 2014 IEEE 15th International Symposium on A World of Wireless, Mobile and Multimedia Networks (WoWMoM), Jun. 2014, pp. 1-6.

[28] A. Kernen, J. Ott, and T. Krkkinen, "The ONE Simulator for DTN Protocol Evaluation," in Proceedings of the $2 \mathrm{Nd}$ International Conference on Simulation Tools and Techniques, ser. Simutools '09. ICST, Brussels, Belgium, Belgium: ICST (Institute for Computer Sciences, Social-Informatics and Telecommunications Engineering), 2009, pp. 55:1-55:10. [Online]. Available: http://dx.doi.org/10.4108/ICST.SIMUTOOLS2009.5674

[29] Q. Li, M. De Rosa, and D. Rus, "Distributed Algorithms for Guiding Navigation Across a Sensor Network," in Proceedings of the 9th Annual International Conference on Mobile Computing and Networking, ser MobiCom '03. New York, NY, USA: ACM, 2003, pp. 313-325. [Online]. Available: http://doi.acm.org/10.1145/938985.939017 